\title{
Two COX-2 inhibitors induce apoptosis in human erythroleukemia K562cells by modulating NF-KB and FHC pathways
}

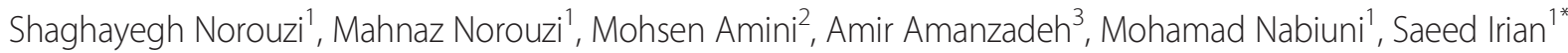
and Mona Salimi ${ }^{4^{*}}$

\begin{abstract}
Background: Leukemia is distinguished by abnormal proliferation of leukocytes. Although there has been some progress in developing novel cancer therapies, no significant improvement was observed in the overall survival rate over the last decade. Selective cyclooxygenase-2 (COX-2) inhibitors are known to inhibit tumor growth by exerting antimetastatic and antiangiogenic effects through inhibition of COX -dependent and independent pathways. The ability of two new triaryl-oxadiazole derivatives, compounds A (3-(4-chlorophenyl) -5-(4-flurophenyl)-4-Phenyl-4,5dihydro-1,2,4-oxadiazole) and B (3,5-bis(4-chlorophenyl)-4-Phenyl-4,5-dihydro-1,2,4-oxadiazole), to induce apoptosis in human erythroleukemia $\mathrm{K} 562$ cells was evaluated and the upstream mechanism was investigated.
\end{abstract}

Methods: K562 cells were treated with compounds A and B at their $\mathrm{I}_{50}$ concentrations and analyzed by DAPI staining and Annexin-V-FLUOS labelling solution. Nuclear factor kappa-B (NF-kB) activation was evaluated by TransAM kit. Cyclooxygenase-2 (COX-2), Caspase-3, Bax, Bcl-2, ferritin heavy chain (FHC), extra cellular signal-regulated kinase (ERK), p-ERK and early growth response protein-1 (Egr1) levels were determined using Western blotting, while c-Myc mRNA level was investigated by RT-PCR.

Results: Changes in nuclear morphology and the increased annexin-V/PI staining revealed the apoptotic cell death in compounds A- and B-treated K562 cells. A significant reduction in NF-KB activity as well as FHC and p-ERK levels were detected in these cells. No change was observed in the levels of Bax, Bcl-2, Caspase-3, COX-2, c-Myc and Egr1, following treatment with the two compounds. Collectively, compounds $\mathrm{A}$ and $\mathrm{B}$ potentiate apoptosis as shown by DAPI staining, flowcytometry, FHC and p-ERK downregulation and NF-KB inactivation.

Conclusion: Two compounds induce apoptosis in a COX-2-independent manner which also appears to be independent from mitochondria, caspase and c-Myc/Egr1 pathways.

Keywords: Leukemia, Apoptosis, COX-2, FHC, NF-kB

\section{Background}

Leukemia, a cancer of the body's blood-forming tissues, including the bone marrow and the lymphatic system, is distinguished by abnormal proliferation of leukocytes. Based on the International Classification of Childhood Cancer, leukemia represents one of the largest diagnostic

\footnotetext{
*Correspondence: irian@khu.ac.ir; salimimona@pasteur.ac.ir; salimi_mona@yahoo.com

'Department of Cell and Molecular Biology, Faculty of Biological Sciences, Kharazmi University, P.O. Box 1481765544 Tehran, Iran

${ }^{4}$ Department of Physiology and Pharmacology, Pasteur Institute of Iran, P.O. Box 13164 Tehran, Iran

Full list of author information is available at the end of the article
}

groups among individuals under 15 years of age with incidence of $34 \%$ [1]. Although there has been some progress in developing novel cancer therapies, no significant improvement was observed in the overall survival rate over the last decade [2]. Nonsteroidal anti-inflammatory drugs (NSAIDs) with their pain relief and anti-inflammation properties have also been the focus of attention as anticancer agents [3]. The targets of traditional NSAIDs are cyclooxygenases 1 and 2 (COX-1 and COX-2), enzymes involved in the production of prostaglandins from arachidonic acid [4]. In this regard, NSAIDs are known to inhibit tumor growth by exerting antimetastatic and 
antiangiogenic effects through inhibition of COX activity, however, a COX-independent pathway has also been suggested $[3,5]$.

In addition to common NSAIDs, the newly developed selective COX-2 inhibitor, celecoxib, with a better gastrointestinal risk profile, has been considered as a costeffective alternative [6]. Celecoxib has been proven as a potent candidate for treating cancer, with several ongoing clinical trials as well as in various animal tumor models [5, 7]. Celecoxib has also been demonstrated to have inhibitory effect on the growth of K562 cells, and induce apoptosis $[5,8]$.

Celecoxib represents a 1,2 -di-aryl heterocyclic structure and used as an ideal lead compound for developing novel derivatives with potent apoptosis-inducing activity $[9,10]$. We have recently reported that two compounds with triaryl-oxadiazole structures known as compounds A (3- (4-chlorophenyl) -5-(4-flurophenyl)-4-Phenyl-4,5dihydro-1,2,4-oxadiazole) and B (3,5-bis(4- chlorophenyl)-4-Phenyl-4,5-dihydro-1,2,4-oxadiazole) (Fig. 1) show significant biological features such as antiproliferative activity with considerable $\mathrm{IC}_{50}$ values $(21.66$ and $22.23 \mu \mathrm{M})$ in human erythroleukemia (K562) cell line after a $24 \mathrm{~h}$ treatment [11]. In the present investigation, we examined the mechanism leading to apoptosis during treatment of K562 cell line with the two new celecoxib derivatives, compounds $\mathbf{A}$ and $\mathbf{B}$.

\section{Methods}

\section{Drugs and reagents}

Compounds A and $\mathbf{B}$ were synthesized by the Department of Medicinal Chemistry, Tehran University of Medical Science (Tehran, Iran). Dulbecco's Modified Eagle's Medium (DMEM) and fetal bovine serum (FBS) were purchased from Gibco-BRL (Rockville, IN, USA). Annexin-V-FLUOS kit was prepared from Roche Applied Science (Indianapolis, USA). Polyclonal anti-caspase-3 (1:500), anti-Bcl-2 (1:500), anti-Bax (1:500), anti-COX-2 (1:1000), anti-GAPDH (1:1000) antibodies and monoclonal anti-ERK (1:1000), anti-Phospho-ERK (1:1000), anti-FHC (1:100) and anti-Egr-1 (1:200) antibodies were purchased from Abcam (Cambridge MA, USA). Anti- rabbit IgG horseradish peroxidase (HRP) antibody (1:5000) was obtained from Cell Signaling Technology (Beverly, MA, USA). All other chemicals were in high purity and prepared from Merck (Darmstadt, Germany).

\section{Cell culture}

K562 cells were obtained from the cell bank of Pasture Institute of Iran (NCBI). Cells were cultured in DMEM medium containing $10 \% \mathrm{FBS}, 100 \mathrm{U} / \mathrm{mL}$ penicillin and $100 \mu \mathrm{g} / \mathrm{mL}$ streptomycin. These cells were incubated at $37{ }^{\circ} \mathrm{C}$ and $5 \% \mathrm{CO} 2$ in a humidified atmosphere and then were treated with compounds $\mathbf{A}$ and $\mathbf{B}$ at the $\mathrm{IC}_{50}$ concentrations $(21.66$ and $22.23 \mu \mathrm{M})$ for 8 and $16 \mathrm{~h}$.

\section{Analysis of cell morphology by DAPI staining}

The treated and untreated cells were stained by DAPI 4,6diamido-2-phenylindole hydro chloride) (Roche Applied Science, Indianapolis, USA), and their morphology was observed under a Zeiss fluorescence microscope (Zeiss, Germany). Photomicrographs were taken with an Olympus digital camera (Tokyo, Japan).

\section{Identification of apoptosis by Annexin-V/PI staining}

Following treatment, $10^{6}$ cells were washed in PBS and resuspended in $100 \mu \mathrm{L}$ of annexin-V-FLUOS labeling solution containing $2 \mu \mathrm{L}$ annexin-V-FLUOS labeling agent, $2 \mu \mathrm{L}$ Propidium Iodide (PI) solution and $1 \mathrm{~mL}$ incubation buffer to achieve a concentration of $10^{6}$ cells $/ \mathrm{mL}$. Following incubation at $37^{\circ} \mathrm{C}$ for 15 minutes, cells were analyzed by flowcytometry. Annexin-V binds to cells expressing phosphatidyl serine on the outer layer of the cell membrane, and PI stains the cellular DNA of those with a compromised cell membrane [12]. This allows for the discrimination of live cells (unstained with either fluorochrome or PI) from apoptotic (stained with annexin-V) and necrotic cells (stained with PI).

\section{Western blotting}

K562 cells were treated with compounds $\mathbf{A}$ and $\mathbf{B}$ at their $\mathrm{IC}_{50}$ concentrations for $16 \mathrm{~h}$. Proteins were extracted from distinctively treated cells, collected and lysed in lysis buffer (Tris $62.5 \mathrm{mM}$ (pH 6.8), DTT 50 mM, SDS
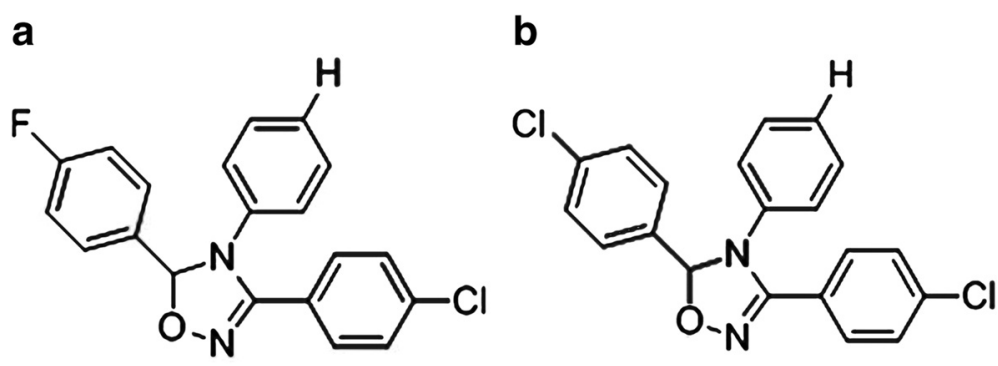

Fig. 1 Structure of the two new celecoxib derivatives 
$10 \%$, glycerol) in the presence of protease inhibitors. Then, equal amounts of protein were heated to $95{ }^{\circ} \mathrm{C}$, separated in $12 \%$ SDS-polyacrylamide gels and transferred to PVDF membranes. The membrane was then

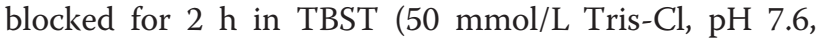
$150 \mathrm{mmol} / \mathrm{L} \mathrm{NaCl}$ and $0.1 \%$ Tween 20) containing $1 \%$ $(\mathrm{w} / \mathrm{v})$ casein, and then incubated with primary antibodies overnight, followed by incubation with HRP conjugated goat anti-rabbit IgG for $2 \mathrm{~h}$. Blots were then developed using ECL advance western blotting detection kit. The signals from each protein band were normalized against the GAPDH (Glyceraldehyde Phosphate Dehydrogenase) content using the polyclonal anti-GAPDH antibody. The expression level of control was designated value " 1 ", and thereby the expression ratios of the treatments were expressed in relation to the control.

\section{ELISA-based TransAM assay}

K562 cells were treated with compounds $\mathbf{A}$ and $\mathbf{B}$ at their $\mathrm{IC}_{50}$ concentrations for $16 \mathrm{~h}$. Proteins of treated cells were extracted. The experiments were performed according to manufacturer's instructions for the ELISAbased TransAM p65 kit (TransAM NF-кB p65 TranscriptionFactor Assay kit). Briefly, $20 \mu \mathrm{g}$ of protein extract per well was added to a 96-well plate coated with the immobilized oligonucleotide containing the NF- $\mathrm{kB}$ consensus sequence (5'-GGGACTTTCC-3') and incubated for $1 \mathrm{~h}$ at room temperature with mild agitation. p65 protein present in the extract will specifically bind to this sequence. Next, anti-p65 antibody $(100 \mu \mathrm{L}$, at a 1:1000 dilution) was added to each well and incubated for $1 \mathrm{~h}$, followed by the addition of $100 \mu \mathrm{L}$ of horseradish peroxidase (HRP)-conjugated antibody (1:1000 dilution) for yet another $1 \mathrm{~h}$. After adding $100 \mu \mathrm{L}$ of the developing solution for up to $5 \mathrm{~min}$, colorimetric reaction was stopped and ELISA reader was used to quantify the difference between the intensity of NF- $\mathrm{kB}$ p65 by reading absorbance at $450 \mathrm{~nm}$ with a correction wavelength of $630 \mathrm{~nm}$.

\section{Semiquantitative RT-PCR}

For analysis of c-Myc mRNA expression levels, cells were treated or not treated with compounds $\mathbf{A}$ and $\mathbf{B}$ for 16 hours, and total RNA was extracted with RNX-Plus (CinnaGen, Iran) following the manufacturer's protocol. $1 \mu \mathrm{g}$ of total RNA was used to perform reverse transcription with the RevertAid first strand cDNA synthesis Kit (Thermo, Lithuania) following the manufacturer's protocol. The resulting reverse transcription products were stored at $-70{ }^{\circ} \mathrm{C}$ until use.

Reaction products were quantified by RT-PCR using a 2X TaqPreMix (Master Mix) PCR Kit (CinnaGen, Iran) according to the manufacturer's instructions. PCR of GAPDH that was chosen as an internal control was carried out in the same tubes as for the genes. The PCR primers of GAPDH and c-myc were synthesized according to the references $[13,14]$. Sequences of the primers were as follows: c-myc (Forward: 5'- TGGTGCTCCATGAGGA GACA-3'; Reverse: 5'-GTGTTTCAACTGTTCTCGTC3'), GAPDH (Forward: 5'-GAGCCCGCAGCCTCCCGCT T-3'; Reverse: 5'- CCCGCGGCCATCACGCCACAG-3').

\section{Statistical analysis}

Each experiment was performed at least three times and representative data were shown. Data in the graph are given as mean values \pm standard error $(\mathrm{SE})$ of the mean. Significant differences among groups were determined using a one-way ANOVA followed by the posttest tukey.

\section{Results}

Apoptosis induction in $\mathrm{K} 562$ cells following compounds A and $B$ treatments

In order to examine whether compounds $\mathbf{A}$ and $\mathbf{B}$ exhibit cytotoxicity in $\mathrm{K} 562$ cells through apoptosis, DAPI staining analysis was undertaken in order to observe morphological changes in K562 cells following treatment with compounds $\mathbf{A}(21.66 \mu \mathrm{M})$ and $\mathbf{B}(22.23 \mu \mathrm{M})$ for 8 and $16 \mathrm{~h}$ (Fig. 2). Morphological evidence of apoptosis was detected as chromatin condensation and nuclear fragmentation following $8 \mathrm{~h}$ (Fig. 2d) and to a greater extent at $16 \mathrm{~h}$ (Fig. 2h) treatment with compound B, whereas compound A-treated K562 cells showed slight morphological changes at $8 \mathrm{~h}$ (Fig. 2c) and to a greater extent at $16 \mathrm{~h}$ (Fig. 2g).

Apoptosis induced by compounds A and B in K562 cells was confirmed by staining cells with Annexin-V/PI and subjecting them to flowcytometry. In the dual parameter fluorescent dot plots, the number of cells in early (annexin $\mathrm{V}+/ \mathrm{PI}-$, the lower right quadrant) and late (annexin $\mathrm{V}+/ \mathrm{PI}+$, the upper right quadrant) apoptosis were counted (Fig. 3). As shown in Table 1, $95.02 \%$ of untreated cells were viable. However, treatment with compounds $\mathbf{A}\left(\mathrm{IC}_{50}\right)$ and $\mathbf{B}\left(\mathrm{IC}_{50}\right)$ for $16 \mathrm{~h}$ resulted in a reduction in the number of viable cells to $90.48 \%$ and $85.95 \%$, respectively, whereas, the percentage of annexin $\mathrm{V}+/ \mathrm{PI}-$ cells (early apoptosis) increased significantly. These results demonstrate that compounds $\mathbf{A}$ and $\mathbf{B}$ show antitumor activities in K562 cells, mainly through the induction of apoptotic cell death.

\section{Effect of compounds A and B on Bax, Bcl-2 and caspase-3 levels}

In order to determine the possible involvement of the caspase cascade in the execution phase of apoptosis, caspase-3 level was investigated following treatment of cells with the $\mathrm{IC}_{50}$ concentration of compounds $\mathbf{A}$ and $\mathbf{B}$ by Western blot analysis. As shown in Fig. 4a, treatment with either compound had no effect on the cleavage of caspase- 3 in $\mathrm{K} 562$ cell line after 8 and 16 h. In addition, 

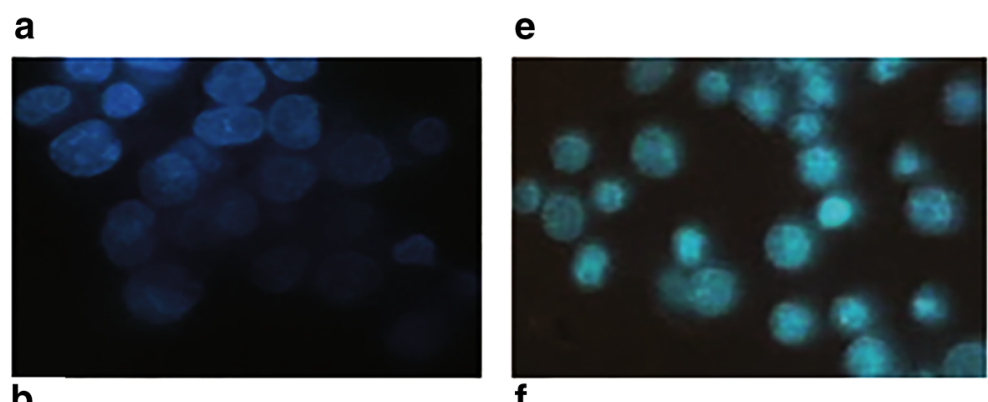

b
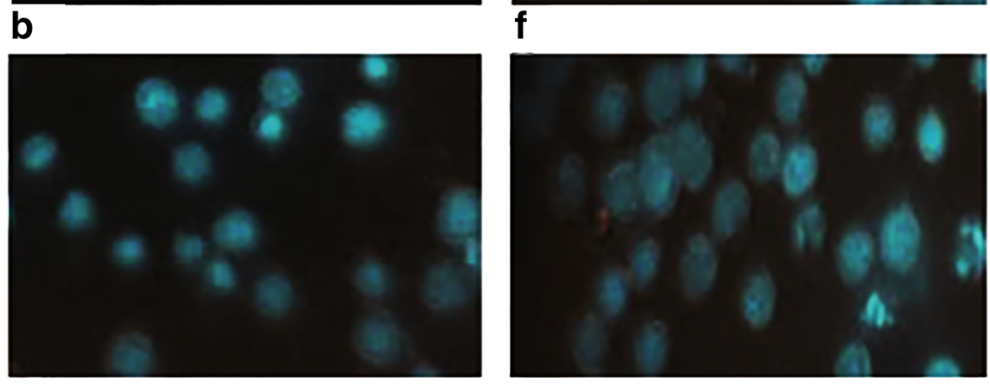

C

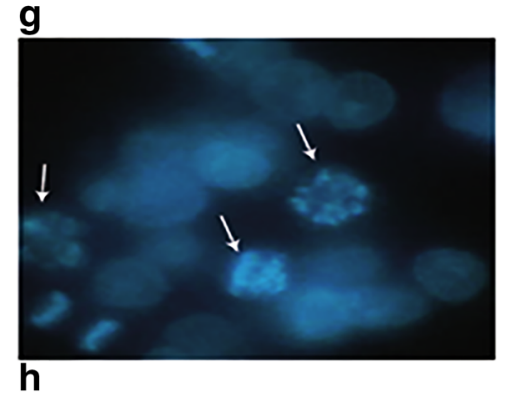

d

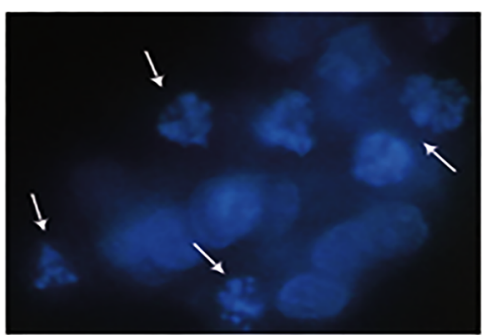

Fig. 2 Apoptotic morphological changes in K562 cells. a K562 control cells after 8 h. b K562 cells treated with DMSO for 8 h. c K562 cells treated with compound A at $21.66 \mu \mathrm{M}$ for $8 \mathrm{~h}$. d K562 cells treated with compound B at $22.23 \mu \mathrm{M}$ for $8 \mathrm{~h}$. e K562 control cells after $16 \mathrm{~h}$. $\mathbf{f}$ K562 cells

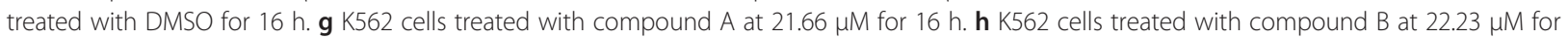
$16 \mathrm{~h}$. Cells were stained with DAPI and observed by fluorescent microscopy

the expression of Bcl-2 and Bax, measured by Western blot analysis, revealed no apparent change in Bax/Bcl-2 ratio at 8 and $16 \mathrm{~h}$ (Fig. $4 \mathrm{~b}$ and c).

\section{Effect of compounds A and B on COX-2 levels}

In order to determine the possible involvement of the COX-2 pathway in apoptosis induced by compounds $\mathbf{A}$ and $\mathbf{B}, \mathrm{COX}-2$ level was investigated following treatment of cells with the $\mathrm{IC}_{50}$ concentrations of the two compounds. As shown in Fig. 5a and b, neither of the compounds had any significant effect on the expression of COX-2 following a $16 \mathrm{~h}$ treatment of K562 cells.
Effect of compounds A and B on NF-KB activity

As NF- $\mathrm{kB}$ plays a critical role in the apoptosis process [15], the effects of compounds $\mathbf{A}$ and $\mathbf{B}$ on NF-kB p65 activity was assessed. As shown in Fig. 6, following a $16 \mathrm{~h}$ treatment of cells with compounds $\mathbf{A}$ and $\mathbf{B}\left(\mathrm{IC}_{50}\right)$, a significant $(p<0.05)$ reduction in the concentration of nuclear p65 subunit was observed.

\section{Effect of compounds A and B on FHC, p-ERK, c-Myc and Egr1 levels}

To reveal upstream mechanism of apoptosis induction following treatment with celecoxib derivatives, levels of certain factors, located downstream of NF- $\mathrm{kB}$ in the 

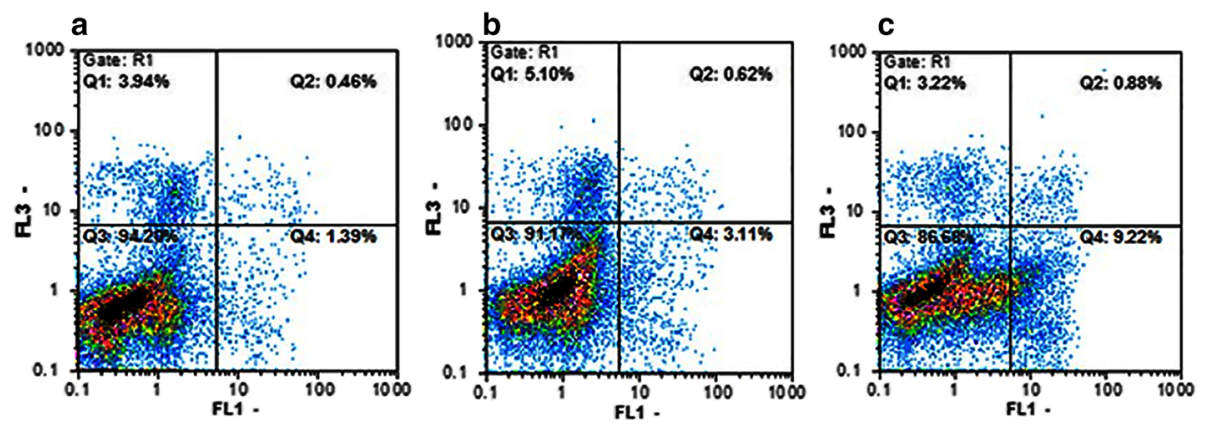

Fig. 3 Flowcytometry analysis to quantify apoptosis in K562 cells. a Untreated control K562 cells. b Cells treated with compound A (21.66 $\mu M$ ). c Cells treated with compound B $(22.23 \mu \mathrm{M})$. Cells were stained with annexin $V$ and propidiumiodide. The results shown are representative of three independent experiments. Quadrant 3, living cells An-/PI-; Quadrant 4, early apoptotic cells An+/PI-; Quadrant 2, late apoptotic cells An+/PI+; Quadrant 1, necrotic cells An-/PI+

apoptosis signaling pathway, including FHC, p-ERK, cMyc and Egr1 were determined through Western and RT- PCR analysis (Fig. 5 and 7).

Administration of compounds $\mathbf{A}$ and $\mathbf{B}$ to $\mathrm{K} 562$ cells resulted in a reduction in FHC protein level, which was significant $(p<0.01)$ following $16 \mathrm{~h}$ of treatment (Fig. 5a and c). Treatment of cells with the two compounds $\mathbf{A}$ and $\mathbf{B}$ also revealed a significant $(p<0.01$ and $p<0.001$, respectively) reduction in the levels of p-ERK which coincided with a lack of change in those of ERK after $16 \mathrm{~h}$ of treatment (Fig. 5a, d and e). Collectively, these results demonstrate that treatment with compounds $\mathbf{A}$ and $\mathbf{B}$ lead to a reduction in both ERK phosphorylation and FHC levels. Finally, Semi-quantitative RT-PCR analysis revealed no change in c-Myc mRNA levels in cells treated with compounds $\mathbf{A}$ or $\mathbf{B}\left(\mathrm{IC}_{50}\right)$ after $16 \mathrm{~h}$ (Fig. 7). Furthermore, consistent with the transcript levels of c-Myc, protein levels of Egr1 were not altered by compounds $\mathbf{A}$ and $\mathbf{B}$ after $16 \mathrm{~h}$ (Fig. $5 \mathrm{a}$ and $\mathrm{f}$ ).

\section{Discussion}

To study the cytotoxicity of compounds $\mathbf{A}$ and $\mathbf{B}$, as two new triaryl-oxadiazole derivatives which well fitted to COX-2 active site [11], their effects on the induction of "programmed cell death" in K562 cells, and the mechanisms by which these compounds induce apoptosis were investigated. K562 cells are a human cancer blood cell line derived from the chronic myeloid leukemia (CML) tumor lineage, which has been considered as a good model to study the cancer inhibitory effects of celecoxib derivatives [5, 8]. Celecoxib, as a first generation of COX-2 inhibitors, has gained a great deal of attention in cancer studies. Although, the cardiotoxic effect has been established in long period treatment of patients with celecoxib, and for this reason, its application in chemoprevention has been restrained [16]. In this regard, the two new compounds $\mathbf{A}$ and $\mathbf{B}$ show significant antiproliferative activity with considerable $\mathrm{IC}_{50}$ values $(21.66$ and $22.23 \mu \mathrm{M})$ in $\mathrm{K} 562$ cell line after a $24 \mathrm{~h}$ treatment [11].

The main mechanism that attributes to the observed effects of COX-2 inhibitors against different types of cancer is apoptosis [17]. Apoptosis is a kind of cell death known as "programmed cell death" that occurs in different physiological and pathological situations and marked by typical morphological and biochemical features, including cell shrinkage, nuclear DNA fragmentation and membrane blebbing [18]. Following treatment of $\mathrm{K} 562$ cells with compounds A and B for $16 \mathrm{~h}$, the fluorescence microscopic observations demonstrated typical morphological changes indicating cell damage; however, these changes were not as much following $8 \mathrm{~h}$ of incubation (Fig. 2). The data obtained from annexin-V/PI analysis were in agreement with the morphological changes showing that compounds $\mathbf{A}$ and $\mathbf{B}$ induce apoptosis after $16 \mathrm{~h}$ (Table 1).

The two major apoptotic pathways known to operate in mammalian cells include the receptor (extrinsic) and the mitochondrial (intrinsic) pathways. Through the intrinsic pathway, members of the $\mathrm{Bcl}-2$ protein family operate as regulators of survival and death during apoptosis induction $[19,20]$. Although the role of

Table 1 Percentage of K562 cells in each state after treatment with compounds A (21.6 $\mu \mathrm{M})$ and B $(22.23 \mu \mathrm{M})$ for $16 \mathrm{~h}$

\begin{tabular}{lllll}
\hline Compound & Vital cells (\%) An-/PI- & Early apoptosis (\%) An+/PI- & Late apoptosis (\%) An+/PI+ & Necrosis (\%) An-/PI+ \\
\hline A & $90.48 \pm 0.38$ & 0.427497 & $0.61 \pm 0.09$ & $4.9 \pm 0.23$ \\
B & $85.91 \pm 0.62$ & $9.82 \pm 0.58$ & $1.05 \pm 0.02$ & $3.22 \pm 0.08$ \\
Control & $95.02 \pm 1.1$ & $1.28 \pm 0.06$ & $0.36 \pm 0.05$ & $3.34 \pm 0.4$ \\
\hline
\end{tabular}




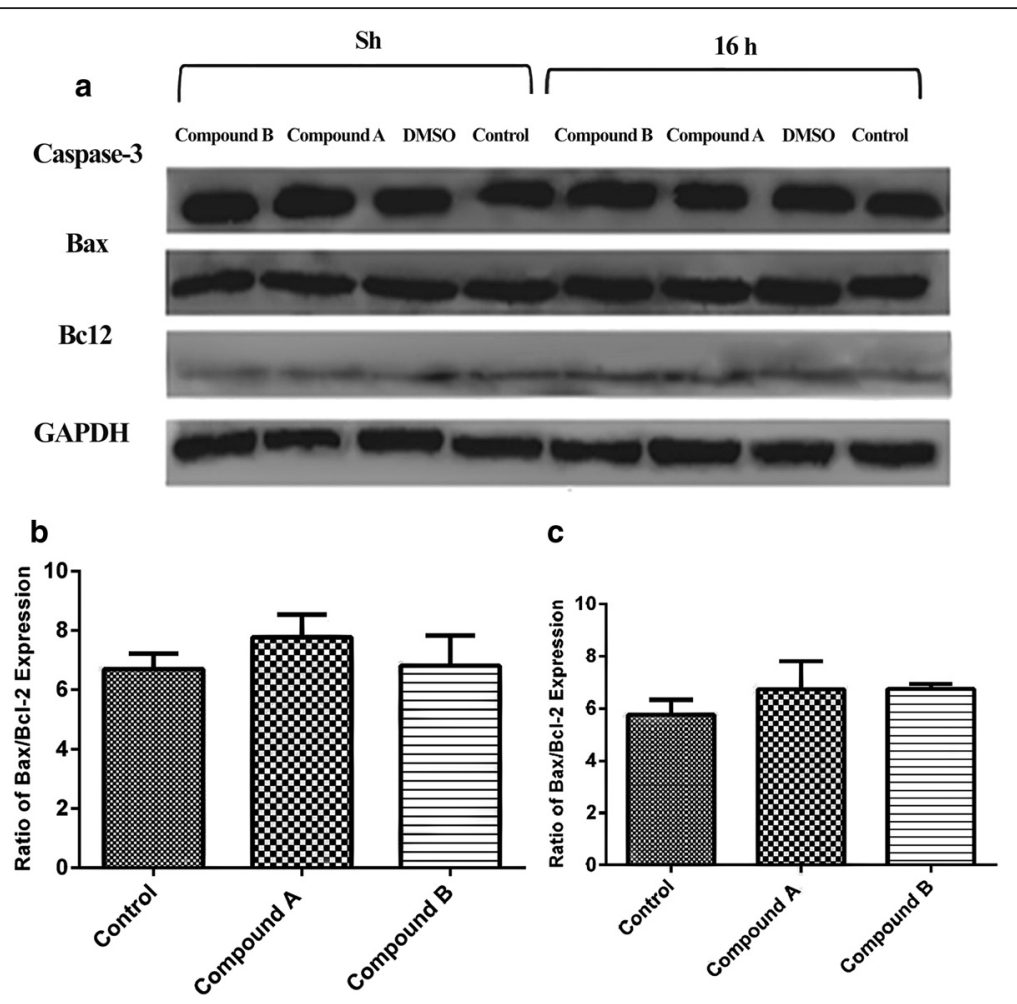

Fig. 4 Expression analysis of apoptosis associated proteins by Western blot analysis. K562 cells were treated with compounds A (21.66 $\mu \mathrm{M})$ and B $(22.23 \mu \mathrm{M})$. a Immunoblot showing the expression levels of caspase-3, Bcl-2, Bax and GADPH. b Ratios of Bax/Bcl-2 after 8 h. c Ratios of Bax/Bcl-2 after $16 \mathrm{~h}$. The results of 3 independent experiments are presented as mean \pm standard error
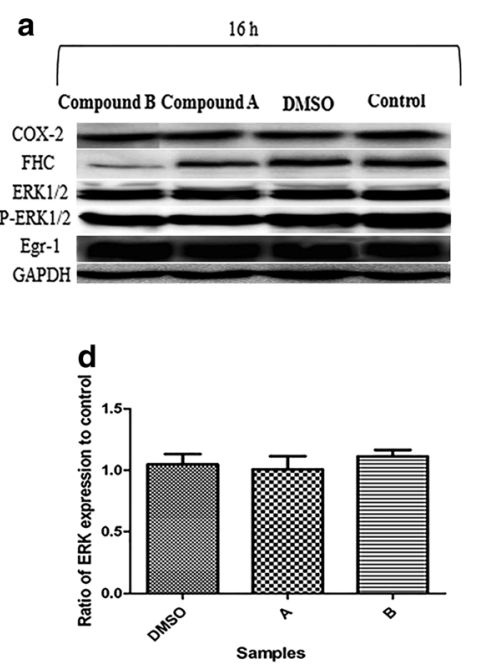
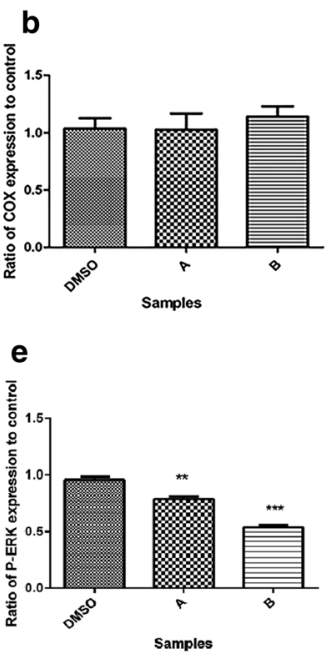
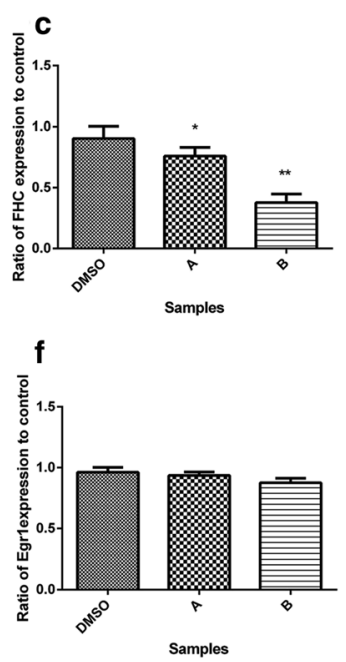

Fig. 5 Effect of compounds A (21.6 $\mu \mathrm{M})$ and $\mathbf{B}(22.23 \mu \mathrm{M})$ on COX-2, FHC, ERK1/2, p-ERK1/2 and Egr1 expression in K562cells after incubation for 16 hours. a Immunoblot showing the expression levels of COX-2, FHC, ERK1/2, p-ERK1/2, Egr1 and GAPDH. b Ratios of COX-2/control. c Ratios of FHC/ control. $\mathbf{d}$ Ratios of ERK/control. e Ratios of p-ERK/control. $\mathbf{f}$ Ratios of Egr1/control. The results of 3 independent experiments are presented as mean \pm standard error $\left({ }^{*} P<0.05,{ }^{* *} P<0.01\right.$ vs. the solvent group) 


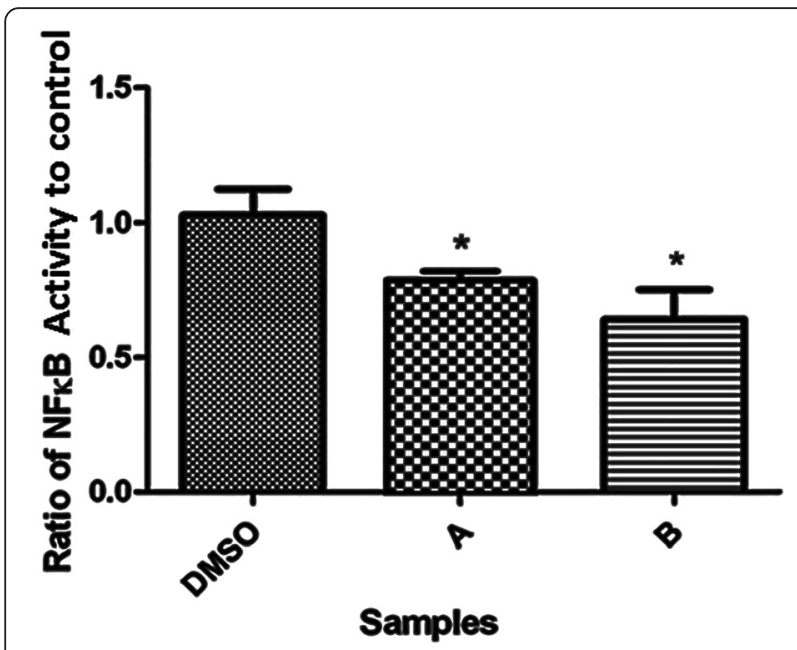

Fig. 6 Effect of compounds $A\left(21.6 \mu M ; I C_{50}\right)$ and $B\left(22.23 \mu M ; I C_{50}\right)$ on NF-kB activation in $\mathrm{K} 562$ cells after incubation for 16 hours. The results of 3 independent experiments are presented as mean \pm standard error ${ }^{*} P<0.05$, vs. the solvent group)

mitochondria in compounds $\mathbf{A}$ - and $\mathbf{B}$-induced apoptosis is not clear in K562 cells, several cytotoxic drugs as well as COX-2 inhibitors are known to initiate apoptosis through the mitochondrial pathway [21]. Our data clearly demonstrate that $\mathrm{Bax}$ and $\mathrm{Bcl}-2$ do not have a critical role in the induction of apoptosis in K562 cells (Fig. 4), suggesting a mitochondria-independent apoptotic pathway induced by compounds $\mathbf{A}$ and $\mathbf{B}$. The role of caspase- 3 in the mitochondria-independent apoptotic pathway has also been well understood [22]. However, apparently compounds $\mathbf{A}$ and $\mathbf{B}$ induce apoptosis in a caspase- independent mechanism as caspase-3 levels were not altered by compounds A and B in K562 cells (Fig. 4a). Collectively these data demonstrate that compounds $\mathbf{A}$ and $\mathbf{B}$ induce apoptosis through both a mitochondria- and caspase- independent mechanism in K562 human leukemia cells. Our obtained data are in agreement with the recent report indicating the execution of apoptosis without the involvement of caspases. Indeed, apoptosis induced in these cells by compounds $\mathbf{A}$ and $\mathbf{B}$ has been shown to involve the activation of proteases other than caspases [23].

The antiproliferative and apoptotic effects of celecoxib in K562 cells are associated with COX-2 inhibition [5]. However, it is well established that tumor growth inhibition by selective COX-2 inhibitors might as well be mediated through COX-2-independent mechanisms [24]. In this study, treatment with compounds $\mathbf{A}$ and $\mathbf{B}$ were found to cause no change in COX-2 levels (Fig. 5). These findings demonstrate that the apoptotic effects of these compounds in $\mathrm{K} 562$ cells are mediated by a COX2 independent pathway. Interestingly, it was recently reported that celecoxib derivatives with no COX-2 inhibitory action can be considered as anti-cancer agents without increasing the cardiovascular risk [25]. Therefore, the two compounds may have a potential in being used as chemotherapeutic agents with little or no cardiovascular side effects.

Induction of apoptosis can be triggered by a number of stimuli, activating a cell suicide program that is constitutively present in most vertebrate cells. In diverse cell types, Rel/NF-kappaB transcription factors are involved in regulation of apoptosis by either inducing or perhaps more commonly blocking it [26]. NF- $\mathrm{kB}$ regulates the expression of more than 400 genes involved in inflammation, cell survival, proliferation, invasion and angiogenesis. NF- $\mathrm{kB}$ also affects apoptosis during tumor development and progression [27], often leading to apoptosis resistance [28]. On the other hand, a reduction in NF- $\mathrm{kB}$ mitogenic signaling has been associated with the anticancer effects of celecoxib treatment on mouse mammary epithelial tumor cells mediated through a COX-2 independent pathway [24]. Our data also demonstrate that treatment of K562 cells with compounds $\mathbf{A}$ and $\mathbf{B}$ results in a reduction of $\mathrm{NF}-\mathrm{\kappa} \mathrm{B}$ expression (Fig. 6) in a COX-2-independent manner.

The primary iron storage factor, FHC (Ferritin heavy chain), which mediates the antioxidant and protective activities of NF- $\kappa \mathrm{B}$, is induced downstream of NF- $\mathrm{B}$ and required for $\mathrm{TNF} \alpha$-induced apoptosis [29]. Rezaie
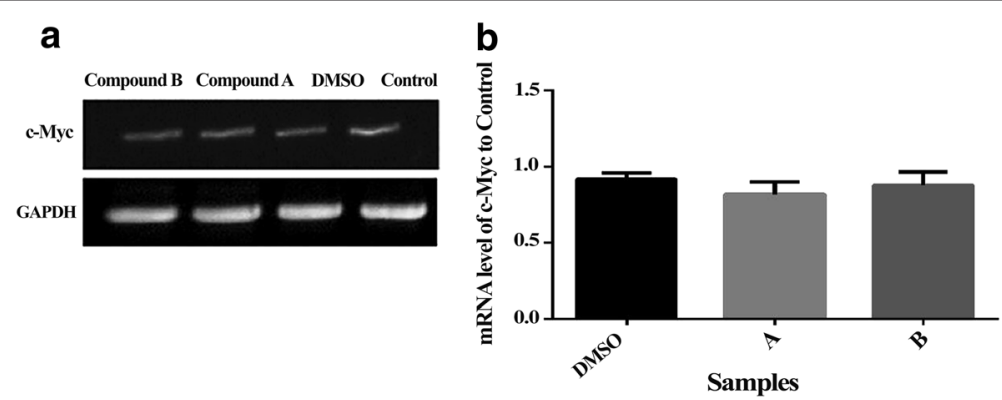

Fig. 7 Effect of compounds $A(21.6 \mu \mathrm{M})$ and B $(22.23 \mu \mathrm{M})$ on c-Myc mRNA levels in K562 cells after incubation for 16 hours. a Immunoblat showing the expression level of c-Myc. $\mathbf{b}$ Ratios of c-Myc/control. The results of 3 independent experiments are presented as mean \pm standard error 
et al. (2011) reported FHC down-regulation in J774.A1 macrophage-like cell lines, following celecoxib treatment [30]. Our study confirms down-regulation of FHC expression induced by compounds A and B in K562 cell line (Fig. 5). Interestingly, our data exhibit the down regulation of both NF- $\mathrm{KB}$ and $\mathrm{FHC}$ which may not be surprising as NF- $\mathrm{kB}$, as a transcription factor, has been involved in the regulation of FHC expression.

Moreover, mitogen-activated protein kinase (MAPK)/ extracellular signal-regulated protein kinase (ERK) have been implied in transmitting the apoptotic signal [14]. MAPK family members are mediators of signal transduction pathways [22]. Importantly, NF-kB acts the upstream of MAPKs in the signaling of certain anticancer drugs [31]. The ERK pathway, a major player in the regulation of cell growth, survival, and differentiation, is induced in response to mitogens and growth factors [22], however, evidence for ERK pathway mediating apoptosis has also been reported [32]. According to previous studies, celecoxib has different roles in ERK1/2 activation in different cells. The present study revealed growth inhibition of K562 cells following compounds Aand $\mathbf{B}$ - treatment via down regulation of p-ERK1/2 (Fig. 5). These data indicate that the mechanism of antiproliferative activities of the designed compounds may be mediated through NF-kB and ERK inhibition.

Anticancer drugs induce apoptosis through different intracellular targets and cause DNA damage [21]. Another factor contributing to the regulation of apoptosis includes the early gene, c-Myc [33]. There has been conflicting reports on the regulation of c-Myc expression during NSAID-induced apoptosis. NSAID-induced apoptosis of gastric cancer cells may be mediated through the upregulation of c-Myc proto-oncogene [34], while NS-398, a COX-2 inhibitor, reduces c-Myc expression in rat colon cancer cells [35]. In the present study, RT-PCR analysis revealed no alteration in the levels of c-Myc transcript in K562 cells treated with compounds A or B compared to the control (Fig. 7b), however, compounds $\mathbf{A}$ and $\mathbf{B}$ induced apoptosis in K562 cells, suggesting the operation of a c-Myc independent apoptotic pathway. Our results are consistent with those of Ostrowski et al. (2003) who demonstrated a lack of alteration in basal and serumstimulated c-Myc expression following treatment of rat hepatoma HTC-IR cells with aspirin or celecoxib. In addition, when treated with the selective COX-2 inhibitor, NS-398, no change was observed in c-Myc mRNA levels in rat colon mucosa as compared to untreated animals [36].

Previous studies also showed that c-Myc directly induces transcription of a noncanonical target gene, Egr1 (Early growth response-1) [37]. Egr1 can suppress the growth of a number of tumor cells, including leukemia. A diverse of anticancer agents, such as COX inhibitors, act through the modulation of Egr1 [38]. In a pancreatic cancer model, tolfenamic acid-induced Egr1 expression appears to have an essential role in the activation of apoptosis [39]. Our data revealed no change in the levels of Egr1 in K562 cell line treated with compounds A and B (Fig. 5). Therefore, the apoptosis in K562 cell line induced by compounds $\mathbf{A}$ and $\mathbf{B}$ appears to be independent from the c-myc/Egr1 pathway. These results are in line with our previous report showing an apoptosis induced by compounds $\mathbf{A}$ and $\mathbf{B}$ via a c-myc/Egr1 independent pathways in breast cancer cell line with a different mechanism [40].

\section{Conclusion}

In summary, the present study reveals that compounds $\mathbf{A}$ and $\mathbf{B}$, as two new triaryl-oxadiazole derivatives with COX-2 inhibitory structures, induce apoptosis in $\mathrm{K} 562$ cells through a mitochondrial-, caspase- and COX-2independent pathways. This COX-2-independent mechanism involves a reduction in NF- $\mathrm{KB}$ levels and a subsequent reduction in the downstream FHC and P-ERK1/2 signaling. Our findings also demonstrate a more pronounced apoptotic effect in response to compound $\mathbf{B}$ than compound A in K562 cells. Future studies need to be directed towards identifying the downstream mediators in order to reveal a more clear and detailed molecular pathway that would help in the development of compounds $\mathbf{A}$ and $\mathbf{B}$ as suitable chemotherapeutic agents against leukemia.

\section{Competing interests}

The authors declare that they have no competing interests.

\section{Authors' contributions}

$\mathrm{SHN}$ and $\mathrm{MN}$ contributed in performing laboratory experiments. MA synthesized the chemical compounds. AA contributed in cell culture techniques. SI and MN contributed in preparing manuscript and the research proposal. MS was the principle investigator who conceived the idea and helped in the preparation of the manuscript. All authors read and approved the final manuscript.

\section{Acknowledgements}

This research was financially supported by Pasteur Institute of Iran and Tehran University of Medical Sciences.

\section{Author details}

${ }^{1}$ Department of Cell and Molecular Biology, Faculty of Biological Sciences, Kharazmi University, P.O. Box 1481765544 Tehran, Iran. ${ }^{2}$ Department of Medicinal Chemistry, School of Pharmacy, Tehran University of Medical Sciences, Tehran, Iran. ${ }^{3}$ National Cell Bank of Iran, Pasteur Institute of Iran, Tehran, Iran. ${ }^{4}$ Department of Physiology and Pharmacology, Pasteur Institute of Iran, P.O. Box 13164 Tehran, Iran.

Received: 3 August 2015 Accepted: 18 December 2015 Published online: 07 January 2016

\footnotetext{
References

1. Anjali P, Faraha S, Amit KS. Synthetic and green vegetable isothiocyanates target red blood leukemia cancers. Fitoterapia. 2012;83:255-65.

2. Shi S, Yao W, Xu J, Long J, Liu C, Yu X. Combinational therapy: New hope for pancreatic cancer. Cancer Lett. 2012;317:127-35.
} 
3. Moon Y, Jr Bottone FG, McEntee MF, Eling TE. Suppression of tumor cell invasion by cyclooxygenase inhibitors is mediated by thrombospondin-1 via the early growth response gene Egr-1. Mol Cancer Ther. 2005;4:1551-8.

4. Hsueh CT, Chiu CF, Kelsen DP, Schwartz GK. Selective inhibition of cyclooxygenase-2 enhances mitomycin-C-induced apoptosis. Cancer Chemother Pharmacol. 2000:45:389-96.

5. Zhang GS, Liu DS, Dai CW, Li RJ. Antitumor effects of celecoxib on K562 leukemia cells are mediated by cell-cycle arrest, caspase-3 activation, and downregulation of Cox-2 expression and are synergistic with hydroxyureaor imatinib. Am J Hematol. 2006:81:242-55.

6. Wang JL, Lin KL, Chen JS, Lu YC, Jiann BP, Chang HT, et al. Effect of celecoxib on $\mathrm{Ca} 2+$ movement and cell proliferation in human osteoblasts. Biochem Pharmacol. 2004;67:1123-30.

7. Pyrko P, Kardosh A, Schönthal AH. Celecoxib transiently inhibits cellular protein synthesis. Biochem Pharmacol. 2008:75:395-404.

8. Subhashini J, Mahipal SV, Reddanna P. Anti proliferative and apoptotic effects of celecoxib on human chronic myeloid leukemia in vitro. Cancer Lett. 2005;224:31-43.

9. Du H, Li W, Wang Y, Chen S, Zhang Y. Celecoxib induces cell apoptosis coupled with upregulation of the expression of VEGF by a mechanism involving ER stress in human colorectal cancer cells. Oncol Rep. 2011;26:495-502

10. Liu X, Yue P, Zhou Z, Khuri FR, Sun SY. Death receptor regulation and celecoxib-induced apoptosis in human lung cancer cells. J Natl Cancer Inst. 2004;96:1769-80

11. Miralinaghi P, Salimi M, Amirhamzeh A, Norouzi M, MostafapourKandelousi $H$, Shafiee A, et al. Synthesis, molecular docking study, and anticancer activity of triaryl-1,2,4-oxadiazole. Med Chem Res. 2013;22:4253-62.

12. Van Engeland M, Nieland $\amalg$, Ramaekers FC, Schutte B, Reutelingsperger CP. AnnexinV affinity assay: a review on an apoptosis detection system based on phosphatidylserine exposure. Cytometry. 1998;31:1-9.

13. Shi $Y$, Hutchinson HG, Hall DJ, Zalewski A. Downregulation of c-myc expression by antisense oligonucleotides inhibits proliferation of human smooth muscle cells. Circulation. 1993;88:1190-5.

14. Sun Y, Li Y, Luo D, Liao DJ. Pseudogenes as weaknesses of ACTB (Actb) and GAPDH (Gapdh) used as reference genes in reverse transcription and polymerase chain reactions. PLoS One. 2012;7:1-11.

15. Fan C, Yang J, Engelhardt JF. Temporal pattern of NFkappaB activation influences apoptotic cell fate in a stimuli-dependent fashion. J Cell Sci. 2002;115:4843-53

16. Benelli R, Venè R, Minghelli S, Carlone S, Gatteschi B, Ferrari N. Celecoxib induces proliferation and Amphiregulin production in colon subepithelialmyofibroblasts, activatingerk1-2 signaling in synergy with EGRF. Cancer Lett. 2013;328:73-82.

17. Cotterchio M, Krieger N, Sloan M, Steinegart A. Non-steroidal anti-inflammatory drug use and breast cancer risk. Cancer Epidemiol Biomarkers Prev. 2001:10:1213-7.

18. Fulda S, Debatin KM. Extrinsic versus intrinsic apoptosis pathways in anticancer chemotherapy. Oncogene. 2006:25:4798-811.

19. Almeida A, Almeida J, Bolanos JP, Moncada S. Different responses of astrocytes and neurons to nitric oxide: the role of glycolytically generated ATP in astrocyte protection. Proc Natl Acad Sci U S A. 2001;98:15294-9.

20. Fujita N, Tsuruo T. Survival-signaling pathway as a promising target for cancer chemotherapy. Cancer Chemother Pharmacol. 2003;1:24-8.

21. Rudner J, Elsaesser SJ, Jendrossek V, Huber SM. Anti-apoptotic BCl-2 fails to form efficient complexes with pro-apoptotic Bak to protect from celecoxib-induced apoptosis. Biochem Pharmacol. 2011;81:32-42.

22. Choi BK, Choi $\mathrm{CH}$, Oh HL, Kim YK. Role of ERK activation in cisplatin-induced apoptosis in A172 human glioma cells. Neuro Toxicology. 2004;25:915-24.

23. Liang Y, Yan C, Schor NF. Apoptosis in the absence of caspase 3. Oncogene. 2001;20:6570-8.

24. Shirode AB, Sylvester PW. Mechanisms mediating the synergistic anticancer effects of combined $\gamma$-tocotrienol and celecoxib treatment. J Bioanal Biomed. 2011:3:1-7.

25. Dovizio M, Tacconelli S, Sostresm C, Ricciotti E, Patrignani P. Mechanistic and pharmacological issues of aspirin as an anticancer agent. Pharmaceuticals. 2012;5:1346-71.

26. Barkett M, Gilmore TD. Control of apoptosis by Rel/NF-kappaB transcription factors. Oncogene. 1999;18:6910-24.

27. Sung B, Pandey MK, Ahn KS, Yi T, Chaturvedi MM, Liu M, et al. Anacardic acid (6-nonadecyl salicylic acid), an inhibitor of histone acetyltransferase, suppresses expression of nuclear factor-kappa B regulated gene products involved in cell survival, proliferation, invasion, and inflammation through inhibition of the inhibitory subunit of nuclear factor-kappaBalph kinase, leading to potentiation of apoptosis. Blood. 2008;111:4880-91.

28. Meteoglu I, Erdogdu IH, Meydan N, Erkus M, Barutca S. NF-KAPPAb expression correlates with apoptosis and angiogenesis in clear cell renal cell carcinoma tissues. J Exp Clin Cancer Res. 2008;27:53-61.

29. Pham CG, Bubici C, Zazzeroni F, Papa S, Jones J, Alvarez K, et al. Ferritin heavy chain upregulation by NFkappa inhibits TNFalpha-induced apoptosis by suppressing reactive oxygen species. Cell. 2004;119:529-42.

30. Rezaie F, Salimi M, Ghahremani MH, Vaziri B. Potential molecular targets in chemopreventative action of celecoxib: a proteomics analysis of J774.A macrophage-like cell line. Mol BioSyst. 2011;7:1306-11.

31. Bava SV, Sreekanth CN, Thulasidasan AK, Anto NP, Cheriyan VT, Puliyappadamba VT, et al. Akt is upstream and MAPKs are downstream of NF-KB in paclitaxel-induced survival signaling events, which are down-regulated by curcumin contributing to their synergism. Int J Biochem Cell Biol. 2001:43:331-41.

32. Zhuang S, Schnellmann RG. A death-promoting role for extracellular signal-regulated kinase. J Pharmacol Exp Ther. 2006;319:991-7.

33. Li Q, Suen TC, Sun H, Arita A, Costa M. Nickel compounds induce apoptosis in human bronchial epithelial Beas-2B cells by activation of c-Myc through ERK pathway. Toxicol Appl Pharmacol. 2009;235:191-8.

34. Zhu GH, Wong BC, Eggo MC, Ching CK, Yuen ST, Chan EY, et al. Non-steroidal anti-inflammatory drug-induced apoptosis in gastric cancer cells is blocked by protein kinase $\mathrm{C}$ activation through inhibition of c-myc Br J Cancer. 1998;79:393-400.

35. Kishimoto Y, Yashima K, Morisawa T, Shiota G, Kawasaki H, Hasegawa J. Effects of cyclooxygenase-2 inhibitor NS-398 on APC and c-myc expression in rat colon carcinogenesis induced by azoxymethane. J Gastroenterol. 2002:37:186-93.

36. Ostrowski J, Wocial T, Skurzak H, Bartnik W. Do altering in ornithine decarboxylase activity and gene expression contribute to antiproliferative properties of COX inhibitors? Br J Cancer. 2003;88:1143-51.

37. Boone DN, Ying Q, Li Z, Hann SR. Egr1 mediates p53-independent c-Myc-induced apoptosis via a noncanonical ARF-dependent transcriptional mechanism. Proc Natl Acad Sci U S A. 2011:108:632-7.

38. Liu J, Liu YG, Huang R, Yao C, Li S, Yang W, et al. Concurrent down-regulation of Egr-1 and gelsolin in the majority of human breast cancer cells. Cancer Genomics Proteomics. 2007:4:377-86.

39. Lee SH, Bahn JH, Choi CK, Whitlock NC, English AE, Safe S, et al. ESE-1/EGR-1 Pathway Plays a Role in Tolfenamic Acid-induced Apoptosis in Colorectal Cancer Cells. Mol Cancer Ther. 2008;7:3739-50.

40. Norouzi M, Norouzi SH, Amini M, Amanzadeh A, Irian S, Salimi M. Apoptotic effects of two COX-2 inhibitors on breast adenocarcinoma cells through COX-2 independent Pathway. J Cell Biochem. 2015;116:81-90.

\section{Submit your next manuscript to BioMed Central and we will help you at every step:}

- We accept pre-submission inquiries

- Our selector tool helps you to find the most relevant journal

- We provide round the clock customer support

- Convenient online submission

- Thorough peer review

- Inclusion in PubMed and all major indexing services

- Maximum visibility for your research

Submit your manuscript at www.biomedcentral.com/submit

\section{) Biomed Central}

